\title{
Psychotherapeutische Diagnostik aus systemischer Perspektive
}

\author{
Elisabeth Wagner
}

Online publiziert: 26. Mai 2020

(C) Der/die Autor(en) 2020

\begin{abstract}
Zusammenfassung Am Anfang des Beitrages wird die traditionell diagnosekritische Haltung der systemischen Therapie dargestellt und theoretisch begründet: sowohl aus der interaktionellen Perspektive der frühen Familientherapie, wie auch aus konstruktivistischer und systemtheoretischer Perspektive wird der Anspruch, intrapsychische Störungen objektiv zu erfassen, in Frage gestellt. Der diagnostische Fokus systemischer (Kurz)Therapie liegt dementsprechend auf der Wirklichkeitskonstruktion der_Klientinnen und Klienten, deren Zielen und Ressourcen und soll eine darauf differenziert abgestimmte therapeutische Kooperation ermöglichen.

In weiterer Folge wird argumentiert, dass trotz aller berechtigter „Diagnoseskepsis“ nicht zuletzt die Ausweitung des Interventionsrepertoires in der systemischen Therapie eine differenziertere Erfassung intrapsychischer Prozesse als Grundlage verantwortungsvollen therapeutischen Handelns erforderlich macht. Diese wird aus einer synergetischen Perspektive dargestellt und der Klassifikation psychischer Störungen nach ICD und DSM gegenübergestellt. Abschließend werden die Dimensionen eines professionellen systemischen Fallverständnisses zusammenfassend dargestellt.
\end{abstract}

Schlüsselwörter Beobachtungen I. und II. Ordnung • Problemkontextualisierung · Interaktionelle Auswirkungen der Symptompräsentation . Ressourcendiagnostik · Synergetisches Verständnis psychischer Prozesse · Dysfunktionale Fühl-DenkVerhaltensprogramme

\footnotetext{
E. Wagner (凶)

Mautner-Markhofstaße 33, 2500 Baden, Österreich wagner.elisabeth@gmx.com
}

\section{Psychotherapeutic diagnostics in the perspective of systemic therapy}

Summary The article begins with the systemic therapy's traditional skepticism towards clinical diagnostics and grounds this theoretically: the standard of being able to objectively asses innerpsychological processes is questioned by being grounded on the interactional perspective of the early family therapy as well as on the constructive and systemic perspective. The systemic short term therapy's focus lays on the client's subjective construction of reality and personal resources and therefore enables a differentiated, individually based therapeutic cooperation.

It is argumented, that despite skepticism towards clinical diagnostics the broadening of interventional repertoire in systemic therapy requires the differentiated assesment of innerpsychological processes, in order to ensure responsible therapeutic actions. This concept is represented by a synergetic perspective and is put into contrast to the classification of psychological disorders as in ICD and DSM.

These dimensions of professional systemic case management and understanding are concluded and summarised.

\section{Die traditionell diagnosekritische Haltung der systemischen (Kurz)Therapie}

Traditionell hat sich die systemische Therapie pointiert diagnosekritisch positioniert. In ihrem Entstehungszusammenhang war dies v.a. durch die interaktionelle Perspektive begründet. Die Pionier_innen der Familientherapie sind angetreten, aktuelle Symptome und Störungen eben nicht durch individuelle Pathologie sondern durch dysfunktionale familiäre Beziehungen zu erklären. Während die frühen Konzepte von Murray Bowen, Lymann Wynne, Theodore 
Lidz u.a. heute nur mehr wenig rezipiert werden, ist die praxisnahe Beschreibung von dysfunktionalen Familienstrukturen durch Salvador Minuchin noch immer nützlich. Sind die Grenzen nach außen und zwischen den Generationen ausreichend klar aber auch flexibel? Gibt es dysfunktionale Koalitionen oder Triangulierungen, die sich auf die kindliche Entwicklung ungünstig auswirken? Strukturelle Familientherapie fokussiert nicht individuelle Pathologie, sondern versucht, die dafür verantwortlichen dysfunktionalen Familienstrukturen zu erfassen und zu verändern.

Erst die konstruktivistische Wende, die in den frühen 80er-Jahren vollzogen wurde, führte $\mathrm{zu}$ einer radikalen Infragestellung von vermeintlich objektiver Erkenntnis und damit auch $\mathrm{zu}$ einer Distanzierung von der Unterscheidung funktional - dysfunktional bzw. normal und gestört. Man verabschiedete sich von dem Glauben, dass Therapeut_innen kraft ihrer Expertise Systemeigenschaften objektiv von außen beurteilen und die entsprechenden Interventionen maßschneidern können. Im Zentrum der modernen Systemtheorie stehen Beobachter_innen, die eine Unterscheidung treffen. Jede Aussage von Beobachter_innen über die Welt ist damit eine Aussage über ihren Akt der Unterscheidung. Aus dieser erkenntnistheoretischen Position konnten nun Krankheiten und Störungen als von „Beobachter_innen“ beobachtete und in sozialer Gemeinschaft erzeugte Phänomene betrachtet werden. Systemische Therapeut_innen interessieren sich daher nicht primär für die „Krankheit an sich“, sondern fokussieren das kommunikative Geschehen rund um diese Beschreibung von Krankheit zwischen Betroffenen, ihren Bezugspersonen und Behandelnden.

Ein weiteres wichtiges Element dieser Beobachter_innen-Theorie ist die Unterscheidung von Beobachtungen I. und II. Ordnung. Beobachtungen I. Ordnung beziehen sich auf die Welt. Beobachtungen II. Ordnung beziehen sich auf Beobachtungen. Die Therapeut_in ist Beobachter_in des Beobachtens: Er_Sie beobachtet, wie Menschen unterscheiden, welche Bedeutungen sie geben, wie sie versprachlichen, welche Geschichten sie über sich und andere erzählen. Damit verschiebt sich der Fokus der Therapie von den äußeren interaktionellen Mustern auf die inneren Prozesse. „An die Stelle des Interesses für Verhaltenssequenzen rückt die Beschäftigung mit der Sprache als basalem Feld für die Konstruktion und Dekonstruktion von Sinn sowie als Rahmen für die Problementwicklung und Problemlösungen“ (Levold 1997). Im Zusammenhang mit der Beobachter_innenTheorie hat die systemische Therapie damit den Anspruch aufgegeben, psychische aber auch interaktionelle Phänomene objektiv beschreiben bzw. mit objektiven Gesetzmäßigkeiten erklären zu können. Nicht nur Diagnosen, sondern alle Beschreibungen und Bewertungen, die Therapeut_innen über ihre Klient_innen anstellen, sind Produkte von Beobachter_innen und müssen nicht hinsichtlich ihrer
„Wahrheit“ sondern hinsichtlich ihrer Nützlichkeit für den therapeutischen Prozess beurteilt werden. Aus dieser theoretischen Position erwuchs ein „konversationales Therapieverständnis“, in dem Interventionen aufgrund ihres Beigeschmacks von Steuerung, außeninduzierter Veränderung und Planbarkeit an Bedeutung verloren und Therapie statt dessen als „Dialog“, „Diskurs“, „Ko-Autorenschaft einer neuen Erzählung“ begriffen wurde.

Jede Art der Diagnostik wird hier als spezifische Wirklichkeitskonstruktion verstanden, die auf ihre Auswirkungen überprüft werden muss. Die Individualisierung und Medikalisierung psychosozialer Probleme wird kritisch gesehen. Systemische Therapeut_innen versuchen daher die in aller Regel problem- und defizitorientierten Merkmale, die durch die Diagnose zugeschrieben werden, zu „verflüssigen“, indem sie in konkretes Verhalten, das zu unterschiedlichen Zeitpunkten in unterschiedlichen Situationen unterschiedlich stark ausgeprägt ist, übersetzt werden. Durch die Kontextualisierung des Symptomverhaltens werden mögliche interaktionale Sinnzusammenhänge sichtbar, durch das Erfassen zirkulärer Ursache-Wirkungs-Beziehungen werden Möglichkeiten der Einflussnahme deutlich.

An diesem Punkt der Theorieentwicklung erreichte die Systemische Therapie die maximale Distanz zu einer klassisch diagnostischen Haltung. Der „diagnostische Fokus“ in der systemischen Kurztherapie ist auf die Wirklichkeitskonstruktion der Klient_innen und auf eine darauf differenziert abgestimmte therapeutische Kooperation, vor allem aber auf den „Möglichkeitsraum“ gerichtet, wie im Folgenden dargestellt werden soll.

\section{Welche diagnostischen Kompetenzen erfordert Systemische (Kurz)Therapie?}

Im Unterschied $\mathrm{zu}$ Therapiemethoden, die vor den eigentlichen Beginn der Behandlung eine diagnostische Phase zur Erarbeitung eines komplexen Fallverständnisses mit daraus folgender Behandlungsplanung stellen, verzichten systemisch arbeitende Therapeut_innen meist auf eine ausführliche anfängliche Exploration der Biographie und der „Störungsanamnese“. Diagnose und Intervention werden nicht als zeitlich oder funktionell getrennte Arbeitsabschnitte gesehen, sondern es werden laufend Informationen gewonnen, die für die Prozess-Steuerung genützt werden.

\section{Problembeschreibung, Problemkontextualisierung, Einstellung zum Problem}

Am Anfang einer systemischen Therapie interessiert sich die Therapeut_in für das Problem, das zur Inanspruchnahme von Therapie führt. Neben dem Selbstverständnis der Klient_in werden auch die Sichtweisen relevanter Anderer, vor allem potenti- 
eller Auftraggeber_innen von Psychotherapie erfragt. Unterschiede in den Sichtweisen und die Bedeutung dieser Unterschiede werden besonders fokussiert. Für systemische Therapeut_innen ist häufig diese „soziale Einbettung“ des Problems von größerer Bedeutung als die detaillierte Symptomschilderung oder die „Krankheitsanamnese“. Symptome beeinflussen Beziehungen, Beziehungen beeinflussen Symptome. Die Bedeutungskonstruktionen rund um das Symptom/Problem bestimmen die Reaktion der Betroffenen und damit die Lebenswirklichkeit der Klient_in. Zirkuläre Fragen zu den Symptomen und ihren Auswirkungen auf Beziehungen, bzw. zu Beziehungen und ihren Auswirkungen auf Symptome zielen auf für Klient_innen relevante Unterschiedsbildungen. Das subjektive Problem/Störungsverständnis der Beteiligten und die Auswirkungen auf das Selbstbild und die Beziehungen bilden damit einen ersten „diagnostischen Fokus“. In diesem Zusammenhang sind auch funktionale Hypothesen möglich: Symptome können eine wichtige, wenn auch häufig unbewusste, interaktionelle Funktionalität aufweisen. Diese kann sichtbar werden, wenn die interaktionellen Auswirkungen der Symptompräsentation erkundet werden.

Einen weiteren wichtigen Fokus stellt die „Einstellung zum Problem“ dar. Steve de Shazer (1989) unterscheidet hier „Besucher“, die subjektiv kein Problem haben, „Klagende“, die unter einem Problem leiden, auf das sie keinen Einfluss haben und „Kunden“, die sich Einfluss auf ihr Problem zuschreiben. In einer lösungsorientierten Haltung ist die subjektive Problemdefinition für das therapeutische Vorgehen bestimmender als eine objektivierende Außenbeschreibung im Sinne einer Störungsdefinition. Klient_innen, die sich Einflussmöglichkeit auf ihre Störungen zuschreiben, behandeln wir ähnlicher, egal, ob es sich dabei um einen Zwang, eine Depression oder Angststörung handelt, als Klient_innen mit der gleichen Störung, die sich diesbezüglich unterscheiden. Für das konkrete therapeutische Vorgehen ist damit nicht primär die Art der Störung ausschlaggebend sondern Faktoren wie Selbstwirksamkeitsüberzeugungen und Veränderungsmotivation, was zum nächsten diagnostischen Fokus - den Zielen und Behandlungserwartungen überleitet.

\section{Ziele und Ressourcen}

Neben dem differenzierten Erfassen des Problemerlebens interessieren sich systemische Therapeut_innen von Anfang an für das „gewünschte Andere“. „Was soll diese Therapie bewirken? Wo wollen Sie hin? Was wäre dann genau anders? Was ist ein erster Schritt in die richtige Richtung?" Durch Fragen wie diese sollen nicht nur positive Behandlungserwartungen induziert werden, sie sollen auch über die Konkretisierung der Vorstellung des „Lösungszustandes“ zur Realisierung zieldienlichen Verhaltens beitragen. Die zugrundeliegende Annahme ist, dass durch die zunehmende kognitive und emotionale Einengung auf das Problem konstruktives Annäherungsverhalten verhindert wird. In dieser Perspektive verweist das Symptom nicht auf ein dahinterliegendes Problem, ihm wird auch keine Funktionalität unterstellt, vielmehr wird die „Problemtrance“ als selbstorganisierte Musterbildung verstanden, die Möglichkeitsräume einengt. Die Konkretisierung und Energetisierung von Annäherungszielen wirkt dieser Einengung entgegen und ist damit ein zentrales Wirkprinzip systemischer Therapie. Die „diagnostische Herausforderung“ (nicht im Sinne einer Störungsdiagnose sondern im Sinne der Prozess-Steuerung) besteht nun darin, jene Ziele zu finden, deren Fokussierung mit positiven somatischen Markern und einer ziel- oder lösungsassoziierten kognitiven Aktivität verbunden ist, damit in einem nächsten Schritt der im Einflussbereich der Klient_in befindliche Beitrag zur Lösung angeregt werden kann. Wenn die Problemtrance nicht wirkungsvoll durchbrochen werden kann, wenn das Reden über das „gewünschte Andere“ keine sichtbaren somatischen Marker und keine lösungsassoziierte kognitive Aktivität auslöst, sondern die Klient_in im leidvollen Erleben verharrt, ist eine strikte Veränderungsorientierung zu diesem Zeitpunkt nicht sinnvoll. In diesen Fällen wird der therapeutische Prozess auf Ressourcenarbeit oder Klärung ausgerichtet. Insofern ist das konkrete therapeutische Vorgehen immer wesentlich davon bestimmt, wo sich die Klient_in in dem Kontinuum zwischen Problemerleben und Realisierung zieldienlichen Verhaltens befindet bzw. in welchem Ausmaß sie sich in Richtung gewünschter Veränderung bewegen kann.

In einem weiteren Verständnis von Diagnostik geht es um das Erfassen aller Faktoren, die für das therapeutische Vorgehen unmittelbar relevant sind. Aus systemischer Perspektive betrifft ein Großteil dieser therapierelevanten Informationen nicht die Störung, sondern das, was für die gewünschte Veränderung nötig ist. Ziele und Ressourcen sind daher für ein systemisches Fallverständnis von besonderer Bedeutung. Welche Ziele sind motivational so hoch besetzt, dass sie als Veränderungsimpulse genützt werden, welche Ressourcen können für diese Veränderung genützt werden? Bei der „Ressourcendiagnostik“ geht es vor allem um positiv besetzte Aspekte der Identität. Über die Fragen: „Worauf sind Sie stolz? Was ist in Ihrem Leben gelungen? Was soll sich nicht ändern? Was würde ihre beste Freund_in sagen, was Sie ausmacht?“ werden Erfahrungen und Kompetenzen sichtbar, die für den Veränderungsprozess nützlich sein können.

\section{Aktuelle Lebenssituation, Genogramm, Entwicklungsaufgaben}

In ein systemisches Fallverständnis fließen immer auch Informationen über die aktuelle Lebenssituation und das soziale Umfeld der Klient_in ein. Das Genogramm bietet einen ersten Überblick über den 
Familienkontext und kann bei Bedarf unter diagnostischer Perspektive genutzt werden. Hinsichtlich der aktuellen Lebenssituation werden nicht nur Belastungsfaktoren und Ressourcen erhoben, sondern auch anstehende „Entwicklungsaufgaben“ fokussiert. Hier bietet z.B. der „Familienlebenszyklus“ (McGoldrick et al. 2009) einen nützlichen Interpretationsrahmen: Die Loslösung vom Elternhaus, das Eingehen einer intimen Partnerschaft, das Elternwerden, und das Heranreifen der Kinder zu Erwachsenen stellen Herausforderungen dar, die für das Fallverständnis diagnostisch genützt werden können. Im familiären Beziehungsgeschehen wird die Fähigkeit entwickelt, in der Interaktion mit anderen Menschen Bindung und Autonomie sowie Grenzen und Austausch angemessen zu gestalten. Dabei auftretende Bindungs-, Ablösungs- und Loyalitätskonflikte, Ausschluss und Ausstoßungserfahrungen können ebenfalls einen „diagnostischen Fokus“ in einem systemischen Fallverständnis bilden.

\section{Bedingungen der Kooperation und „Reagibilität“}

Für die Prozess-Steuerung in Systemischen Therapien ist eine genaue Beobachtung der Klient_innen in Hinblick auf ihr Verhalten in der Therapie unerlässlich. Als zentrale Denkfigur würde ich in diesem Zusammenhang nicht Übertragungsphänomene sondern „Reagibilität“ vorschlagen. In Analogie zu Kurt Lewins Äußerung, wonach man ein soziales System erst versteht, wenn man versucht, es zu verändern gilt auch für psychische Systeme, dass sich in der Reaktion auf Fragen und Anregungen des Therapeuten wichtige „diagnostische“ Informationen finden. Welchen Anregungen zur Aufmerksamkeitsfokussierung folgt die Klient_in? Welche Ideen und Unterschiedserzeugungen sind anschlussfähig? Wie ist die aktuelle „Arbeitshaltung“ oder Kooperationsfähigkeit der Klient_in einzuschätzen? Befindet sich die Klient_in in einem produktiven Prozess der Auseinandersetzung mit den anstehenden Problemen? Nützt sie_er das therapeutische Gespräch, um ihren_seinen Einfluss auf die Zielerreichung, das heißt ihre_seine Selbstwirksamkeit zu erhöhen? Wenn nicht: welche berücksichtigungswürdigen Bedürfnisse müssen vielleicht vorher erfüllt werden?

Das konkrete therapeutische Vorgehen ist so zu gestalten, dass Klient_innen bei der konstruktiven Auseinandersetzung mit aktuellen Anforderungen und anstehenden Entwicklungsschritten optimal unterstützt werden (vgl. Wagner 2020, S. 223). Hierzu gehört auch die Wahl des geeigneten Settings („Wer könnte Teil der Lösung sein und etwas zur Verbesserung der Situation beitragen?“) und eine adäquate „Problemformatierung“: Aus konstruktivistischer Perspektive ist dabei zu berücksichtigen, dass wir mit unseren Vorannahmen und Fragen mit darüber entscheiden, welche Sachverhalte als zum Problem gehörend (eingeschlossen) und welche als nicht zum Problem ge- hörend (ausgeschlossen) betrachtet werden. Ob wir bei einer Anorexie, einer Angststörung oder einer depressiven Störung frühe Beziehungserfahrungen biographie- und problemorientiert fokussieren, ob wir eine funktionale Hypothese bezüglich der aktuellen Lebenssituation formulieren („Für welches Problem ist das präsentierte Symptom die Lösung?“) oder in einem lösungsorientierten Therapieverständnis die Aufmerksamkeit auf die Ausnahmen und Ressourcen lenken, ist eine therapeutische Entscheidung, die fallbezogen getroffen und verantwortet werden muss. „Es ist eine therapeutische Entscheidung, wie das Problem formatiert wird. Die zentrale Operation dieser therapeutischen Entscheidung ist Einschluss (gehört dazu) oder Ausschluss (hat nichts damit zu tun) bestimmter Sachverhalte. Wenn Probleme komplexitätserhöhend und hochinklusiv beschreiben werden (vieles, gehört dazu'), wird eine lange Dauer nahegelegt, weil mehr Fragen behandelt und beantwortet werden müssen“ (vgl. Fischer et al. 2015, S. 45).

\section{„Diagnostizieren“ auf der Basis eines synergetischen Verständnisses psychischer Prozesse}

Systemische (Einzel-)Therapie wird in den letzten Jahren zunehmend unter synergetischer Perspektive beschrieben (Schiepek et al. 2013; Grossmann 2014; Wagner et al. 2016; Kriz 2017). Psychische Prozesse werden dabei unter Bezugnahme auf Luc Ciompi als jeweils spezifische Fühl-Denk-Verhaltens-Muster konzeptualisiert und rücken in den Fokus des therapeutischen Interesses. Dies ist vor allem deshalb relevant, weil sich unter dem Einfluss von Aufstellungsarbeit, Teilearbeit und hypnosystemischen Ansätzen das Interventionsrepertoire der systemischen Therapie in den letzten Jahren merklich ausgeweitet hat. Es werden zunehmend auch affektaktualisierende und erlebnisorientierte Interventionen eingesetzt, durch die spezifische Erfahrungen angeregt werden sollen. Hier verlassen wir das Interventionsterrain konversationaler konstruktivistischer (oder auch narrativ-lösungsorientierter) Therapie, was auch eine Ausweitung der theoretischen Grundlagen um eine differenzierte Konzeptualisierung psychischer Prozesse erfordert (vgl. Wagner und Russinger 2016). Störungen der Emotionsverarbeitung, die Vermeidung schmerzhafter Emotionen aber auch strukturelle Defizite können damit für das konkrete Fallverständnis Bedeutung erlangen.

Die differenzierte Erfassung und Beschreibung dysfunktionaler psychischer Prozesse ist bislang keine Domäne systemischer Therapie. Auf der Basis einer synergetischen Konzeptualisierung ist sie allerdings möglich, wie im Folgenden kurz dargestellt werden soll. In einem synergetischen Verständnis sind psychische Vorgänge affektiv-kognitive Prozesse, eine Abfolge von sich wiederholenden bzw. sich selbst organisierenden Operationen. Das aktuelle Erleben 
ist das Ergebnis selbstorganisierter Musterbildung neuronaler Aktivität und wird durch Bahnungsphänomene und die aktuelle Aufmerksamkeitsfokussierung beeinflusst. Grossmann (2014) unterscheidet in diesem Zusammenhang zwischen prozeduralem und narrativem Selbst. Das prozedurale Erleben „ereignet“ sich, spontanes Erleben kann aber durch Selbstreflexion bewusst wahrgenommen und in weiterer Folge modifiziert werden.

Zentral ist hier der von Luc Ciompi $(1997,2002)$ eingeführte Begriff der Fühl-Denk-Verhaltensprogramme (FDV-Programm), der weite Überlappungen mit dem Schemabegriff in der Psychologie aufweist. Heute versteht man unter einem Schema die neuronal verankerte Reaktionsbereitschaft, aktuelle Situationen vor dem Hintergrund früherer Erfahrungen zu interpretieren. Wahrnehmung, Empfindung, Bedeutung und Handlungsimpuls entstehen spontan, ein FühlDenk-Verhaltensprogramm wird „hochgefahren“. Unter günstigen Bedingungen entwickelt sich ein flexibles Potential an funktionalen FDV-Programmen, was dazu beiträgt, dass wichtige Grundbedürfnisse befriedigt werden können. Je belastender die Entwicklungsbedingungen sind, desto größer ist aber die Wahrscheinlichkeit, dass sich dysfunktionale FDVProgramme entwickeln.

Im Hinblick auf diagnostische Anforderungen verweist eine systemtheoretische Sicht zunächst darauf, dass die Psyche kein beobachtbares Etwas mit beständiger Struktur darstellt, das sinnvollerweise anhand beobachtbarer Muster und Eigenschaften beschrieben werden könnte. Psychische Phänomene stellen Ereignisse oder Ereignisfolgen dar, die genau wie Kommunikation, nicht zur dinglichen Welt gehören. Psychische Phänomene sind damit aus systemtheoretischer Sicht Prozesse, die sich autonom und selbstorganisiert auf der Basis eigener elementarer Operationen der Sinnstiftung und Sinnbewahrung bilden und durch Reproduktion für ihre Kontinuität sorgen. „Der Verzicht auf unpassend gewordene, verdinglichende Metaphern des Psychischen als Apparat ... muss nicht bedeuten, dass man ganz und gar auf strukturelle Aspekte verzichten muss“ (Ludewig 2013, S. 204). In dem Bemühen, „die Lücke zwischen einer theoriekonformen abstrakten Betrachtung sozialer und psychischer Systeme und den konkreten Bedürfnissen von Psychotherapeuten zu schließen“ (Ludewig 2013, S. 208), kann es sinnvoll sein, störungsspezifische Konzepte zu nützen, um jene dysfunktionalen Muster der psychischen Selbstorganisation berücksichtigen zu können, die das leidvolle Erleben mitverursachen oder aufrechterhalten.

Hier geht es allerdings nicht um die nosologische Perspektive von ICD und DSM, die impliziert, dass psychische Störungen klar getrennte Entitäten sind, die mit der gleichen Exaktheit zu klassifizieren sind wie Carl Linné das Pflanzen- und Tierreich geordnet oder Mendelejew das Periodensystem der Elemente definiert hat. Ein Symptom, eine Störung oder
Krankheit sind aus systemtheoretischer Sicht eben keine Dinge, die nach bestimmten Prinzipien geordnet werden können, sondern Prozesse, gebildet durch Wahrnehmungsmuster, Erlebnisweisen, Bedeutungskonstruktionen, Handlungen und Kommunikationen verschiedener Personen.

Die Kritik an der Pseudoobjektivierung psychischer Störungen durch moderne Diagnoseschemata ist keine genuine Leistung systemischer Therapie. Auch von anderen Therapiemethoden und selbst innerhalb der Psychiatrie wird von mehreren Seiten der deskriptive Ansatz, d.h. die Klassifikation psychischer Störungen auf der Basis beschreibbarer Symptome in Frage gestellt. Von neurowissenschaftlicher Seite wird argumentiert, dass eine rein syndromorientierte Klassifikation zwangsläufig zu einer fehlerhaften Nosologie psychischer Krankheiten führt, da sie verschiedene Ursachen, die in ähnlichen Syndromen resultieren, gleich klassifiziere. Dementsprechend fordert auch das National Institute of Mental Health (NIMH) mit seiner Research Domain Criteria Initiative (RDoC), die Klassifikation psychischer Störungen dem in der Medizin dominierenden kausalen Krankheitsbegriff stärker anzupassen und die zugrunde liegenden neurobiologischen Eigenschaften zu berücksichtigen (vgl. Walter und Müller 2015).

Von phänomenologischer Seite wird der Versuch, durch psychiatrische Klassifikationssysteme „die schwer greifbaren seelischen Erlebnisse gewissermaßen zu materialisieren, sie dingfest und manipulierbar zu machen“ (Fuchs 2015, S. 29) ebenfalls ganz grundsätzlich kritisiert. Reduktionismus, Verdinglichung und Medikalisierung tragen dazu bei, das Leid der Betroffenen getrennt von den Wechselwirkungen mit ihrer_seiner Umgebung zu betrachten, aus denen heraus das leidvolle Erleben entstanden ist und mit aufrechterhalten wird. Thomas Fuchs fordert daher, neben dem positivistischen, objektivierenden Ansatz von DSM und ICD (3. Person-Perspektive), sich auch dem subjektiven Selbsterleben der Patient_in zuzuwenden (1. Person-Perspektive) und ihr_sein Selbstkonzept, ihre_seine persönliche Geschichte, ihre_seine Beziehungsmuster, Motive und Konflikte in einer gemeinsamen Konstruktion von Sinn und Bedeutung $\mathrm{zu}$ erfassen (2. Person-Perspektive). Dies allerdings funktioniert nicht mit Symptomchecklisten sondern nur über den aufwändigen Weg von Verstehen und Einfühlen.

Die Auseinandersetzung mit subjektiven Sinnzusammenhängen und Bedeutungskonstruktionen, mit individuellen Erlebnisweisen und Motivationsdynamiken ist für die psychotherapeutische Praxis konstitutiv. Um die Klient_in in ihrer_seiner Auseinandersetzung mit ihrem_seinem Gewordensein, ihren_seinen Ängsten, Schwierigkeiten, Sehnsüchten und Zielen zu unterstützen, kann aber auch die Erfassung dysfunktionaler psychischer Prozesse, die am leidvollen Erleben beteiligt sind, nötig sein. Auf diesem Weg werden Klient_innen dabei unterstützt, dysfunktionale Fühl- 
Denk-Verhaltens- und damit auch Interaktionsmuster zu erkennen, zu benennen und zu verändern.

Auch wenn wir psychische Störungen als selbstorganisierte Reproduktion biographisch entstandener dysfunktionaler Muster begreifen, sind Kausalkonstruktionen im Sinne eines „weil damals, deshalb heute“ aus systemischer Perspektive kritisch zu betrachten. Nicht das Erlebte per se, sondern die darauf folgende Musterbildung bzw. fortbestehende Dominanz eines (Erlebnis)Musters sind „Ursache“ des aktuellen Problems: „Im Unterschied zu Kausalkonstruktionen, die Gegenwärtiges kausal auf Früheres zurückführen, ist aus systemtheoretischer Sicht die ,Wiederholung' die ,Ursache'. Vergangenheit überlebt demnach ,Wiederholungsakte“" (Lieb 2014, S. 68). In der therapeutischen Kooperation können solche Wiederholungen identifiziert und als Wiederholungen markiert werden und es kann überlegt werden, wie die erneute Wiederholung unterbrochen werden kann. Allerdings erweisen sich biographisch gewachsene und durch unzählige Wiederholungen gut gebahnte FDV-Programme häufig als resistent gegenüber kurztherapeutischen Methoden und erfordern Erweiterungen des typisch systemischen Interventionsrepertoires, wie sie im Buch „Emotionsbasierte Systemische Therapie. Intrapsychische Prozesse verstehen und behandeln“ (Wagner und Russinger 2016) dargestellt wurden.

Die erste diagnostische Einschätzung betrifft die Frage, ob und welche Muster der intrapsychischen Selbstorganisation am leidvollen Erleben beteiligt sind und gezielt adressiert werden müssen, um die Veränderung in die von der Klient_in gewünschte Richtung zu ermöglichen. Neben dysfunktionalen Schemata sind es vor allem Störungen der emotionalen Verarbeitung und Hinweise auf strukturelle Störungen, die in einem differenzierten Fallverständnis berücksichtigt werden müssen. Dabei ist zunächst eine grundlegende Unterscheidung zu treffen: Handelt es sich um eine stabile Klientin_einen stabilen Klienten (evtl. emotional überreguliert), bei der_dem das Erleben von Emotionen aktiviert und gefördert werden soll oder um eine instabile, impulsive Klientin_einen instablien, impulsiven Klienten, die_der Unterstützung bei der Emotionskontrolle braucht? Die Entscheidung, ob emotionales Erleben aktiviert und als Informationsquelle über nicht realisierte Grundbedürfnisse genützt wird oder aber Kontrolle über dysfunktionale heftige Emotionen und damit eine Distanzierung von überbordenden Gefühlen gefördert wird, ist für das weitere therapeutische Vorgehen von zentraler Bedeutung.

In diesem Zusammenhang ist auch die Unterscheidung von primären und sekundären Emotionen (vgl. Greenberg 2011) hilfreich, bei der davon ausgegangen wird, dass die primäre situationsadäquate Emotion (z. B. Ärger) nicht wahrgenommen werden kann, weil sie sich vor dem Hintergrund der jeweils individuellen Lerngeschichte als unerwünscht, gefährlich, etc. erwiesen hat. An ihre Stelle tritt eine sekundäre Emotion (z.B. Traurigkeit), die dann zunehmend generalisieren kann. Auch dieses Phänomen hat Eingang in die moderne systemtheoretisch fundierte Therapie gefunden: „Das Konzept der Unterscheidung von primären und sekundären Gefühlen hält hierzu ein weiteres nützliches Element bereit: die klinisch oft bestätigte Annahme, dass ein psychisches System, das in sich primäre Gefühle wie Schmerz, Trauer oder Wut erfährt, keine weiteren Operationen einsetzen muss, um diese Gefühle wieder zu verändern. Sie hören von selbst auf - im Unterschied zu sekundären Gefühlen, die sich aufgrund ihrer Regenerierung durch Operationen der Psyche selbst immer wieder aufbauen“ (Lieb 2014, S. 91). Therapeut_innen sollten daher nachfragen, welche Emotionen in welchen Situationen erlebt werden, um dann Hypothesen darüber bilden zu können, ob die Emotionswahrnehmung eingeschränkt ist, ob sekundäre Emotionen das Erleben dominieren und damit den Blick auf die wesentlichen Bedürfnisse verstellen (vgl. Wagner und Russinger 2016, S. 142).

Nach den dysfunktionalen FDV-Programmen und den Störungen der Emotionsverarbeitung sollte auch das „Funktionsniveau der Persönlichkeit“, wie es im Anhang des DSM-5 ausgeführt wird, diagnostisch erfasst werden. Hier schließt moderne psychiatrische Diagnostik erstmals an die lange Tradition struktureller Überlegungen der Psychoanalyse an. Strukturelle Fähigkeiten dienen zur Regelung des inneren Gleichgewichts und der gelebten Beziehungen. Dementsprechend erhebt die Level of Personality Functioning Scale Beeinträchtigungen in je zwei Dimensionen des Selbst (Identität und Selbststeuerung) und der interpersonellen Beziehungen (Empathie und Nähe). Für die Erfassung dieser strukturellen Fähigkeiten muss die Therapeut_in die von der Klient_in formulierten konkreten Themen, Probleme und Anliegen vorübergehend defokussieren und statt dessen versuchen, die „Funktionsweise des Ichs“ im Längsschnitt zu erfassen.

Die Therapeut_in nimmt damit eine Beobachter_innen-Haltung hinsichtlich der psychischen Funktionen ein: Gibt es ein kohärentes Bild von sich selbst und wichtigen anderen, kann die_der Betroffene selbstreflexiv innerseelische Vorgänge wahrnehmen? Kann sie_er ihre_seine Affekte differenziert wahrnehmen und in Sprache fassen? Können auch unangenehme Affekte erlebt und adäquat ausgedrückt werden? Kann sie_er mit anderen in Kontakt treten und sich in deren Innenwelt einfühlen, kann sie_er in nahen Beziehungen für Interessensausgleich sorgen? Kann sie_er sich emotional an andere binden und diesen gegenüber Dankbarkeit, Fürsorge oder Schuld erleben? Kann sie_er Trennungen tolerieren und adäquat verarbeiten? (vgl. Wagner und Russinger 2016, S. 129).

Therapeut_innen sollten eine „handlungsleitende Vorstellung“ darüber entwickeln, ob dysfunktionale 
Schemata, strukturelle Defizite oder andere Störungen der Emotionsverarbeitung (z. B. Emotionsvermeidung oder ein emotionsphobischer Konflikt) wesentlich an der Aufrechterhaltung der Leidenszustände beteiligt sind. Die Erfassung dieser problematischen Aspekte der Selbstorganisation ist allerdings keine autoritäre Expertenleistung, vielmehr werden durch einen gemeinsamen Such- und Erkundungsprozess die dysfunktionalen Muster, die dem symptomatischen Erleben zugrunde liegen oder es aufrechterhalten, identifiziert und angesprochen. Auf diese Weise wird klar, welche Problemdeterminanten im Augenblick für die Klient_innen anschlussfähig und damit für Interventionen zugänglich sind.

\section{Dimensionen eines systemischen Fallverständnisses}

Im Unterschied zur psychiatrischen Diagnose organisiert das Fallverständnis Informationen über die psychosoziale Bedingungskonstellation, über die subjektiven Überzeugungen genauso wie über objektivierbare Symptome und Defizite, biographische Belastungen und aktuelle Anforderungen der Lebenssituation. Manche systemische Kolleg_innen sprechen lieber von „Fallverstehen“, weil damit noch deutlicher wird, dass es sich dabei um einen bewussten, interpretierenden, hermeneutischen Prozess handelt. Fallverstehen ist immer vorläufig und muss sich im therapeutischen Handeln bewähren, indem es dieses begründet. Es ist immer das Ergebnis von gezieltem Explorationsverhalten aber auch Selektionsleistungen der Therapeut_in. In das Fallverständnis fließen in der Regel Informationen aus folgenden Bereichen ein:

a) „soziale Identität“: Alter, Geschlecht, Zugehörigkeit zu sozialer Schicht, Familiensituation, nahe Beziehungen, berufliche Identität, evtl. Ethnie, Religion, sexuelle Orientierung, Weltanschauung

b) das präsentierte Problem (Konsultationsgrund, Anlass) und die interaktionellen Muster rund um das Problem

c) die Beziehung zum Problem, das Narrativ über das Problem, subjektives Problemverständnis auch von relevanten Anderen, „Krankheitstheorie“ aber auch Veränderungsmotivation (vgl. Besucher, Klagender, Kunde bei de Shazer (1989))

d) die Beziehung zur Therapeut_in (Kooperation, Vertrauen ...), Kontaktverhalten in der Therapie, Erwartung an die Therapie

e) evtl. „Geschichtlichkeit“ - wichtige biographische Faktoren, was ist gelungen, was nicht, welche Entwicklungsschritte stehen an unter Berücksichtigung des Familienlebenszyklus nach Carter und McGoldrick (1980), sowie des entwicklungspsychologischen Modells von Erik Erikson (1992)

f) Erfassung von Potentialen und Ressourcen, evtl. Werthaltungen, Ideale, die für die Veränderungsmotivation hilfreich sein können g) Erfassung aktueller Belastungsfaktoren (ökonomische Lage, gesundheitlicher Zustand ...)

h) sofern vorhanden: die psychiatrische Störung in ihrer Eigendynamik (z. B. bipolare Störung, psychotische Störungen, Substanzstörungen, PTBS,..)

i) sofern vorhanden: problematische Aspekte der psychischen Selbstorganisation oder spezifische Symptome, die das therapeutische Arbeitsbündnis oder die Zielerreichung limitieren (Selbstverletzung, Aggressivität, Impulsivität, Substanzabusus ...)

- dysfunktionale Schemata und Überzeugungen

- Störungen der Emotionsverarbeitung

- strukturelle Defizite

Aus systemischer Perspektive ist die Therapeut_in dafür verantwortlich, durch die Exploration aber auch Selektion relevanter Informationen ein Fallverständnis zu entwickeln, das sie_ihn handlungsfähig macht und auf der Basis dieses Fallverständnisses die selbstreflexiven Prozesse der Klient_in in einer Weise anzuregen, dass ihre_seine Selbststeuerung ziel- und bedürfniskonform besser gelingt. Die Therapeut_in entscheidet darüber, wohin sie mit ihren_seinen Fragen den Erzählfluss und damit die Aufmerksamkeit lenkt, welche Bedeutungen sie_er anbietet und welche konkreten Anregungen sie_er für neue Erfahrungen (innerhalb und außerhalb der Therapiesituation) formuliert (vgl. Weber 2012). Systemische Therapeut_innen gehen aber nicht davon aus, dass in einer konkreten therapeutischen Situation nur eine einzige Vorgehensweise, nur ein einziges Fallverständnis „richtig“ und erfolgsversprechend ist. Sowohl die Entstehungszusammenhänge von Problemen und Störungen als auch deren Überwindung können immer unterschiedlich interpretiert werden. Das konkrete Fallverständnis dient der Komplexitätsreduktion und soll hilfreiches Intervenieren ermöglichen. Es ist daher nicht am Wahrheitskriterium sondern an seiner Nützlichkeit zu messen. Die Konsensualisierung des Fallverstehens zwischen Therapeut_in und Klient_in ist dabei ein wesentliches und für Systemische Therapie konstitutionelles Merkmal im diagnostischtherapeutischen Prozess: Ein „richtiges“ - im Sinne eines angemessenen - Fallverständnisses ist immer das Ergebnis einer Übereinkunft in Bezug auf die Antwort auf die Frage „wie können WIR verstehen, was hier der Fall ist".

Interessenkonflikt E. Wagner gibt an, dass kein Interessenkonflikt besteht.

Open Access Dieser Artikel wird unter der Creative Commons Namensnennung 4.0 International Lizenz veröffentlicht, welche die Nutzung, Vervielfältigung, Bearbeitung, Verbreitung und Wiedergabe in jeglichem Medium und Format erlaubt, sofern Sie den/die ursprünglichen Autor(en) und die Quelle ordnungsgemäß nennen, einen Link zur Creative Commons Lizenz beifügen und angeben, ob Änderungen vorgenommen wurden. 
Die in diesem Artikel enthaltenen Bilder und sonstiges Drittmaterial unterliegen ebenfalls der genannten Creative Commons Lizenz, sofern sich aus der Abbildungslegende nichts anderes ergibt. Sofern das betreffende Material nicht unter der genannten Creative Commons Lizenz steht und die betreffende Handlung nicht nach gesetzlichen Vorschriften erlaubt ist, ist für die oben aufgeführten Weiterverwendungen des Materials die Einwilligung des jeweiligen Rechteinhabers einzuholen.

Weitere Details zur Lizenz entnehmen Sie bitte der Lizenzinformation auf http://creativecommons.org/licenses/by/4. 0/deed.de.

\section{Literatur}

Carter, E.A., \& McGoldrick, M. (1980). The Family life cycle: a framework for family therapy. NewYork: Gardner Press.

Ciompi, L. (1997). Die emotionalen Grundlagen des Denkens. Entwurf einer fraktalen Affektlogik. Göttingen: Vandenhoeck\& Ruprecht.

Ciompi, L. (2002). Gefühle, Affekte, Affektlogik. Wien: PicusVerlag.

De Shazer, S. (1989). Der Dreh. Überraschende Wendungen und Lösungen in der Kurzzeittherapie. Heidelberg: Carl Auer.

Erikson, E. H. (1992). Der vollständige Lebenszyklus. Frankfurt: Suhrkamp.

Fischer, H.R., Borst, U., \& v. Schlippe, A. (2015). Was tun? Fragen und Antworten aus der systemischen Praxis. Ein Kompass für Beratung, Coaching und Therapie. Stuttgart: Klett-Cotta.

Fuchs, T. (2015). Subjektivität und Intersubjektivität. Zur Grundlage psychiatrischer und psychotherapeutischer Diagnostik. Kontext, 46, 27-41.
Greenberg, L.S. (2011). Emotionsfokussierte Therapie. München: Reinhardt.

Grossmann, K. (2014). Systemische Einzeltherapie. Göttingen: Hogrefe.

Kriz, J. (2017). Subjekt und Lebenswelt. Personzentrierte Systemtheorie für Psychotherapie, Beratung und Coaching. Göttingen:Vandenhoeck\&Ruprecht.

Levold, T. (1997). Affekte und System. System Familie, 10, $120-127$.

Lieb, H. (2014). Störungsspezifische Systemtherapie. Konzepte und Behandlung. Heidelberg: Carl Auer.

Ludewig, K. (2013). Entwicklungen systemischer Therapie. Einblicke, Entzerrungen, Ausblicke. Heidelberg: CarlAuer.

McGoldrick, M., Giordano, J., \& Petri, S. (2009). Genogramme in der Familienberatung (3. Aufl.). Bern:Huber.

Schiepek, G., Eckert, H., \& Kravanja, B. (2013). Grundlagen systemischer Therapie und Beratung. Psychotherapie als Förderung von Selbstorganisationsprozessen. Göttingen: Hogrefe.

Wagner, E. (2020). Praxisbuch Systemische Therapie. Vom Fallverständnis zum wirksamen therapeutischen Handeln in klinischen Kontexten. Stuttgart: Klett-Cotta.

Wagner, E., \& Russinger, U. (2016). Emotionsbasierte systemische Therapie. Intrapsychische Prozesse verstehen und behandeln. Stuttgart:Klett-Cotta.

Wagner, E., Henz, K., \& Kilian, H. (2016). Persönlichkeitsstörungen. Störungen systemisch behandeln. Heidelberg: CarlAuer.

Walter, H., \& Müller, J. (2015). Der Beitrag der Neurowissenschaften zum psychiatrischen Krankheitsbegriff. Nervenarzt, 86, 22-28.

Weber, R. (2012). Navigieren auf Sichtweite - Prozesssteuerung in der Paartherapie. Stuttgart: Klett-Cotta.

Hinweis des Verlags Der Verlag bleibt in Hinblick auf geografische Zuordnungen und Gebietsbezeichnungen in veröffentlichten Karten und Institutsadressen neutral. 